\title{
Electron and photon beams interacting with plasma
}

\author{
By Albert Reitsma and Dino Jaroszynski \\ University of Strathclyde, Department of Physics \\ John Anderson Building, 107 Rottenrow, Glasgow G4 ONG, UK
}

A comparison is made between the interaction of electron bunches and intense laser pulses with plasma. The laser pulse is modelled with photon kinetic theory, i.e. a representation of the electromagnetic field in terms of classical quasi-particles with space and wave number coordinates, which enables a direct comparison with the phase space evolution of the electron bunch. Analytical results are presented of the plasma waves excited by a propagating electron bunch or laser pulse, the motion of electrons or photons in these plasma waves and collective effects, which result from the self-consistent coupling of the particle and plasma wave dynamics.

Keywords: Beam-plasma interaction; laser-plasma interaction; advanced accelerators

\section{Introduction}

Plasma is an attractive medium for acceleration of charged particles (Esarey et al. 1996) due to its ability to sustain large accelerating gradients without suffering from electrical breakdown, which is a major limiting factor in conventional accelerators. Suitable drivers for high-amplitude plasma waves are dense, ultra-short relativistic electron bunches (Chen et al. 1985) or intense laser pulses (Tajima \& Dawson 1979), which are sufficiently powerful to induce charge separation in plasma and set up a travelling space charge wave or wakefield behind themselves. Understanding the evolution of these wakefield drivers as well as the evolution of witness beams, i.e. trailing beams to be accelerated in the plasma wave, is key to developing plasmabased accelerators. The dynamics of intense beams in plasma is complicated due to the relativistic, nonlinear plasma response and the occurrence of instabilities (Keinigs \& Jones 1987; Guérin et al. 1995), both of which require fully self-consistent modelling.

The evolution of laser pulses and electron bunches has similarities and differences, and these are best understood by treating electrons and photons on equal footing. This can be done in an elegant way by using photon kinetic theory (Silva \& Mendonça 1998), a classical particle description of the photons. The main idea behind photon kinetic theory is to split a laser pulse into quasi-particles that obey the ray-tracing equations of motion, which model the adiabatic changes in wave number and frequency due to the space and time variations of the permittivity of the plasma, and group velocity dispersion effects due to finite bandwidth. The quasi-particles are best thought of as 'dressed' particles that represent the collective behaviour of photons in a plasma. A Vlasov equation for the quasi-particle phase 
space density can be formally derived from Maxwell's equations by assuming that the plasma permittivity varies on space and time scales that are much longer than the laser wavelength and period, respectively. This is usually the case for laser pulse propagation in underdense plasma. The representation of a laser pulse with space and wave number coordinates enables a direct comparison with the phase space evolution of a bunch of relativistic electrons.

In this paper we introduce all the aspects of the interaction step by step, restricting ourselves to one-dimensional geometry, as the length of this paper does not allow a full discussion of three-dimensional aspects of the problem. We begin with the plasma response to a given driver, which can be either a laser pulse or an electron bunch, in $\S 2$. Usually the driver sets up a wakefield, so we proceed with describing electron and photon motion in wakefields in $\S 3$. We then introduce distribution functions to calculate the wakefield sources in a self-consistent way, so that feedback from the particles on the plasma wave is taken into account. This gives rise to collective effects, two examples of which will be described in $\S 4$. The first example is beam loading, i.e. the effect that a witness beam takes energy from a given wakefield by setting up its own plasma wave that (partly) cancels the wake wave. The second example is the evolution of a short driver in its own wakefield. A summary and discussion of the results is offered in $\S 5$.

\section{Wakefields}

In this section, we describe the travelling space charge waves or wakefields driven by a laser pulse or relativistic electron bunch that propagates in a plasma. For the plasma electron dynamics, we use the cold fluid model in the quasi-static approximation (Ting et al. 1990). Plasma ions are assumed to be stationary and to provide a neutralizing background with density $n_{0}$, which is used to define the ambient plasma frequency $\omega_{p}$ by the relation $\omega_{p}^{2}=4 \pi n_{0} e^{2} / \mathrm{m}$. The continuity equation for the plasma electron density $n(z, t)$ is $\partial n / \partial t+\partial\left(n v_{z}\right) / \partial z=0$, where $v_{z}=p_{z} / \gamma m$ is the longitudinal velocity, $p_{z}(z, t)$ the longitudinal momentum and $\gamma(z, t)$ the Lorentz factor, which contains a contribution from the quiver motion in the laser field $\gamma^{2}=1+p_{z}^{2} / m^{2} c^{2}+e^{2} A_{\perp}^{2} / m^{2} c^{4}$, as follows from identifying the perpendicular momentum $\vec{p}_{\perp}=e \vec{A}_{\perp} / c$ with the vector potential $\vec{A}_{\perp}$ that describes the electromagnetic fields of the laser pulse. The equation for the longitudinal momentum is $\partial p_{z} / \partial t=e \partial \varphi / \partial z-m c^{2} \partial \gamma / \partial z$, where $\varphi$ denotes the electrostatic potential. The $\partial \gamma / \partial z$-term represents the ponderomotive force, while the $\partial \phi / \partial z$-term is the electrostatic field. The fluid model is completed with Poisson's equation $\partial^{2} \varphi / \partial z^{2}=4 \pi e\left(n+n_{b}-n_{0}\right)$, where $n_{b}(z, t)$ is the density of the relativistic electron bunch. The quasi-static approximation consists of replacing the full $(z, t)$-dependence of all plasma quantities with a dependence on $\zeta=z-c t$ only. This means that each element of the plasma electron fluid responds in the same way to the driver of the plasma wave as it passes by. This approximation is only valid if changes to $A_{\perp}^{2}$ or $n_{b}$ can be neglected during the time it takes the laser pulse or electron bunch to propagate a distance equal to its own length, i.e. if the driver evolves slowly in the co-moving frame. The quasi-static approximation leads to the relation $e \varphi=(\gamma-1) m c^{2}-c p_{z}$, which enables all plasma quantities to be expressed in terms of $A_{\perp}^{2}$ and $\varphi$, for example $\gamma=\left[1+\phi+\left(1+a^{2}\right) /(1+\phi)\right] / 2$, where the poten- 
tials have been cast in dimensionless form $\left(\phi=e \varphi / m c^{2}, \vec{a}=e \vec{A}_{\perp} / m c^{2}\right)$. Usually, the plasma is taken to be unperturbed $\left(p_{z}=0, n=n_{0}\right)$ in front of the wakefield driver. An important quantity is the space and time-varying plasma frequency $\Omega_{p}$, which takes into account relativistic and ponderomotive effects:

$$
\Omega_{p}^{2}=\frac{4 \pi n e^{2}}{\gamma m}=\frac{\omega_{p}^{2}}{1+\phi} .
$$

Due to the identification $\partial / \partial \zeta=\partial / \partial z=-(1 / c) \partial / \partial t$, the fluid model reduces to a single wakefield equation

$$
\frac{\partial^{2} \phi}{\partial \xi^{2}}=\frac{1}{2}\left[\frac{1+a^{2}}{(1+\phi)^{2}}-1\right]+\frac{n_{b}}{n_{0}}
$$

where a dimensionless coordinate $\xi=\omega_{p} \zeta / c$ has been introduced. In source-free regions, i.e. where $a=0$ and $n_{b}=0$, the wakefield equation has an invariant (Teychenné et al. 1994)

$$
\mathcal{C}=\frac{1}{2}\left[\left(\frac{\partial \phi}{\partial \xi}\right)^{2}+\frac{\phi^{2}}{1+\phi}\right]=\frac{E_{0}^{2}}{2}
$$

where $E_{0}$ is the amplitude of the dimensionless electric field $\partial \phi / \partial \xi$. From equation (2.3) it is straightforward to derive expressions for the extrema $\phi_{ \pm}$of the potential in terms of $E_{0}$

$$
1+\phi_{ \pm}=1+E_{0}^{2} / 2 \pm E_{0}\left(1+E_{0}^{2} / 4\right)^{1 / 2}=\left[E_{0} / 2 \pm\left(1+E_{0}^{2} / 4\right)^{1 / 2}\right]^{2},
$$

which will be used in some of the calculations presented below.

\section{Equations of motion}

In this section, we present the equations of motion for electrons and photons that interact with wakefields. In the previous section, we introduced the coordinate $\zeta=z-$ ct that moves with the speed of light in vacuum. In this section, it is useful to redefine this coordinate $\zeta=z-v_{r} t$ as moving with the phase velocity of the wakefield, which we call the resonant velocity $v_{r}$. For calculating the shape of the wakefield, as done above, the approximation $v_{r} \approx c$ is allowed. However, for calculating the motion of accelerated electrons or photons, the difference between $v_{r}$ and $c$ is important to account for phase slippage with respect to the wakefield. It is convenient to define the resonant Lorentz factor $\gamma_{r}=\left(1-\beta_{r}^{2}\right)^{-1 / 2}$, with $\beta_{r}=v_{r} / c$. It is straightforward to derive the electron equations of motion

$$
\frac{d \zeta}{d t}=\frac{P_{z}}{\gamma m}-v_{r}, \quad \frac{d P_{z}}{d t}=e \frac{\partial \varphi}{\partial \zeta}-\frac{e^{2}}{2 \gamma m c^{2}} \frac{\partial A_{\perp}^{2}}{\partial \zeta},
$$

from a Hamiltonian $\mathcal{H}_{e}=\gamma m c^{2}-v_{r} P_{z}-e \varphi$ (Reitsma et al. 2001) with canonical coordinate and momentum variables $\left(\zeta, P_{z}\right)$. Note that here the notation $\gamma$ is used for the Lorentz factor of an individual electron with coordinates $\zeta(t)$ and $P_{z}(t)$, rather than for a field $\gamma(z, t)$ that depends on space and time. 

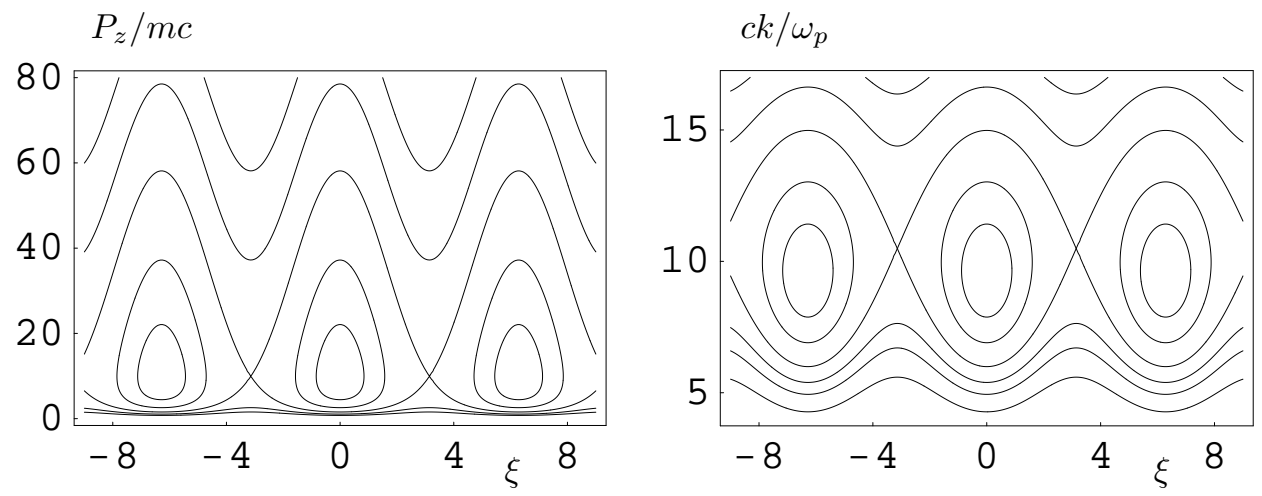

Figure 1. Phase diagrams for dynamics in a plasma wave, where the left hand side plot shows electron orbits and the right hand side plot shows photon orbits. The dimensionless plasma wave amplitude is $E_{0}=0.1$ and the resonant Lorentz factor is $\gamma_{r}=10$.

Using the solution for $\phi$ found in the previous section, we can construct a phase diagram for the Hamiltonian $\mathcal{H}_{e}$ (Esarey \& Pilloff 1995). As seen in Fig. 1, there are closed orbits inside the separatrix and open orbits both above and below the separatrix. The orbits below the separatrix describe the motion of electrons that are too slow to be captured in the wave, e.g. the orbit corresponding to the plasma electron fluid motion. The orbits above the separatrix correspond to the motion of electrons that are outrunning the wave. The orbits inside the separatrix describe the motion of electrons that are trapped inside the wave. For orbits inside the separatrix, one defines the turning points by the condition $d \zeta / d t=0$ : at these points the backward phase slip of the electron changes to forward slip or vice versa. As seen in Fig. 1, these points are found at $P_{z} / m c=\beta_{r} \gamma_{r}$. Points of minimum and maximum energy, defined by $d P_{z} / d t=0$, are found at $\phi=\phi_{ \pm}$. Equilibrium points are found where $d \zeta / d t=0$ and $d P_{z} / d t=0$ simultaneously, with $\phi_{+}$corresponding to stable equilibrium (O-points) and $\phi_{-}$corresponding to unstable equilibrium (Xpoints). The value of $P_{z}$ on a particular orbit with $\mathcal{H}_{e}=h_{e} m c^{2}$ at a particular phase, characterized by $\phi=\phi_{0}$, is given by

$$
P_{z} / m c=\beta_{r} \gamma_{r}^{2}\left(h_{e}+\phi_{0}\right) \pm \gamma_{r}\left[\gamma_{r}^{2}\left(h_{e}+\phi_{0}\right)^{2}-1\right]^{1 / 2} .
$$

In particular, the separatrix corresponds to $h_{e}=1 / \gamma_{r}-\phi_{-}$and the maximum and minimum values of $P_{z}$ on the separatrix, denoted $P_{ \pm}$, correspond to $\phi_{0}=\phi_{+}$. It is interesting to evaluate $P_{ \pm}$in the regime of high plasma wave amplitude $\gamma_{r} \Delta \phi \gg 1$

$$
P_{+} / m c \approx 2 \gamma_{r}\left(1+\gamma_{r} \Delta \phi\right), \quad P_{-} / m c \approx(1 / \Delta \phi-\Delta \phi) / 2
$$

where $\gamma_{r} \gg 1$ has been used, and $\Delta \phi$ is short for $\phi_{+}-\phi_{-}=E_{0}\left(4+E_{0}^{2}\right)^{1 / 2}$. At $\Delta \phi>1$, which corresponds to $E_{0}>\left(5^{1 / 2}-2\right)^{1 / 2} \approx 0.486$, it is found that $P_{-}<0$. The results in the low plasma wave amplitude regime $\gamma_{r} \Delta \phi \ll 1$ are

$$
P_{ \pm} / m c \approx \gamma_{r}\left[\beta_{r} \pm\left(2 \gamma_{r} \Delta \phi\right)^{1 / 2}\right] .
$$

As mentioned in the introduction, for describing the motion of photons in plasma waves we use the ray-tracing equations

$$
\frac{d \zeta}{d t}=\frac{c^{2} k}{\Omega}-v_{r}, \quad \frac{d k}{d t}=-\frac{1}{2 \Omega} \frac{\partial \Omega_{p}^{2}}{\partial \zeta},
$$


which are derived from a Hamiltonian $\mathcal{H}_{p}=\Omega-v_{r} k$. The dependence of the frequency $\Omega$ on the phase space coordinates is given by a local dispersion relation $\Omega^{2}=c^{2} k^{2}+\Omega_{p}^{2}$. The identification of wave number with momentum and frequency with energy establishes the similarity between the dynamics of electrons and photons in plasma waves and explains the use of the terms 'photon acceleration' and 'photon deceleration' for the frequency upshift, respectively downshift of photons. The effective mass $m_{p}$ of a photon in a plasma, given by $m_{p}=\hbar \Omega_{p} / c^{2}$, is seen to depend on the position of the photon in the wave due to the dependence of $\Omega_{p}$ on $\zeta$. The equivalent of $\gamma$ is $\Omega / \Omega_{p}$, which also depends on the position of the photon in the wave.

Using the solution for $\phi$ found in the previous section, we can construct a phase diagram for $\mathcal{H}_{p}$ (Mendonça \& Silva 1994) in the same way as we did for $\mathcal{H}_{e}$. As seen in Fig. 1, the same classification of orbits (inside, below or above the separatrix) applies to the photon Hamiltonian. The points of maximum and minimum frequency are given by the condition $d k / d t=0$, corresponding to $\phi=\phi_{ \pm}$, which is equal to the condition for points of maximum and minimum electron energy found above. The turning points, defined by $d \zeta / d t=0$, are found at $c k / \Omega_{p}=\beta_{r} \gamma_{r}$. Interestingly, the value of $k$ depends on the phase of the wave due to the $\zeta$-dependence of $\Omega_{p}$. This is in contrast with the electron case, where the value of the momentum at the turning point $P_{z} / m c=\beta_{r} \gamma_{r}$ does not depend on $\zeta$. The value of $k$ on a particular orbit with $\mathcal{H}_{p}=h_{p} \omega_{p}$ at a particular phase, given by $\phi=\phi_{0}$, is given by

$$
c k / \Omega_{p}=\beta_{r} \gamma_{r}^{2} h_{p}\left(1+\phi_{0}\right)^{1 / 2} \pm \gamma_{r}\left[\gamma_{r}^{2} h_{p}^{2}\left(1+\phi_{0}\right)-1\right]^{1 / 2}
$$

The separatrix corresponds to $\gamma_{r} h_{p}=\left(1+\phi_{-}\right)^{-1 / 2}$ and the minimum and maximum wave number on the separatrix are denoted $k_{ \pm}$. In the limit of high plasma wave amplitude $1+\phi_{+} \gg 1$ or $E_{0} \gg 1 / 4$, the expressions for $k_{ \pm}$are

$$
c k_{+} / \omega_{p} \approx 2 \gamma_{r}\left(1+\phi_{+}\right)^{1 / 2}, \quad c k_{-} / \omega_{p} \approx\left[\gamma_{r} /\left(1+\phi_{+}\right)^{3 / 2}-\left(1+\phi_{+}\right)^{1 / 2} / \gamma_{r}\right] / 2
$$

where $\gamma_{r} \gg 1$ has been used. The condition for $k_{-}<0$ is given by $1+\phi_{+}>\gamma_{r}$, corresponding to $E_{0}>\gamma_{r}^{1 / 2}-\gamma_{r}^{-1 / 2}$. Finally, in the low plasma wave amplitude limit $\Delta \phi \ll 1$ it is found that

$$
c k_{ \pm} / \omega_{p} \approx \gamma_{r}\left[\beta_{r} \pm(\Delta \phi)^{1 / 2}\right]
$$

\section{Collective effects}

The motion of a group of electrons or photons, i.e. the evolution of an electron bunch or laser pulse, is conveniently expressed in a distribution function $f_{b}\left(P_{z}, \zeta, t\right)$ for the electron bunch or $f_{p}(k, \zeta, t)$ for the laser pulse. The distribution function obeys a Vlasov equation, namely

$$
\frac{\partial f_{b}}{\partial t}+\frac{\partial f_{b}}{\partial \zeta} \frac{\partial \mathcal{H}_{e}}{\partial P_{z}}-\frac{\partial f_{b}}{\partial P_{z}} \frac{\partial \mathcal{H}_{e}}{\partial \zeta}=0
$$

for the electron bunch and

$$
\frac{\partial f_{p}}{\partial t}+\frac{\partial f_{p}}{\partial \zeta} \frac{\partial \mathcal{H}_{p}}{\partial k}-\frac{\partial f_{p}}{\partial k} \frac{\partial \mathcal{H}_{p}}{\partial \zeta}=0
$$


for the laser pulse. To model collective effects, one needs a self-consistent description of the interaction of electrons and photons in plasma, which is obtained when the Vlasov equations are coupled to the wakefield equation by deriving the wakefield sources from the distribution functions. The electron bunch density is calculated from the electron bunch distribution by integration over the momentum variable

$$
n_{b}(\zeta, t)=\int f_{b}\left(P_{z}, \zeta, t\right) d P_{z},
$$

while the spatial envelope of the vector potential involves a weighting with $1 / \Omega$ as follows

$$
a^{2}(\zeta, t)=c \int \frac{f_{p}(k, \zeta, t)}{\Omega(k, \zeta, t)} d k .
$$

Below we present two examples of collective effects.

\section{(a) Beam loading}

Beam loading (Wilks et al. 1987) is the effect that a witness beam takes energy from a given plasma wave by setting up its own wakefield, i.e. a second plasma wave that (partly) cancels the first wave. For the moment, we neglect the evolution of the first wave due to the feedback from the plasma on the drive beam.

\section{(i) Electrons}

Let the witness be an electron bunch, which we will take to be infinitely short $n_{b}(\xi) / n_{0}=q_{b} \delta\left(\xi-\xi_{b}\right)$, where $q_{b}$ denotes the dimensionless charge of the bunch and $\xi_{b}$ the position of the bunch in the plasma wave. The rate of energy transfer from the plasma wave to the bunch is equal to the difference across the bunch of the wakefield invariant $\mathcal{C}$ defined in equation (2.3). The dimensionless electric field $\partial \phi / \partial \xi$ makes a jump of magnitude $q_{b}$ across the bunch, from which the magnitude of the jump in $\mathcal{C}$ is calculated to be $\Delta \mathcal{C}=q_{b} \partial \phi / \partial \xi\left(\xi_{b}\right)-q_{b}^{2} / 2$. Thus the electric field acting on the bunch is effectively $\partial \phi / \partial \xi-q_{b} / 2$, i.e. the sum of the prescribed field and a constant decelerating field due to the bunch's own wakefield. If the sign of $\Delta \mathcal{C}$ is positive, i.e. if $\partial \phi / \partial \xi\left(\xi_{b}\right)>q_{b} / 2$, then the bunch gains energy from the wave. If $q_{b}>2 E_{0}$, the bunch can not take energy from the wave at any phase. For calculating the total energy transferred from the wave to the bunch, one needs to know the time dependence of $\xi_{b}$, i.e. the phase slippage of the electron bunch. In the limit $\gamma \gg \gamma_{r}$, one may take $v_{z}=c$ for all bunch electrons. which leads to $d \xi_{b} / d \tau \approx 1-\beta_{r} \approx 1 /\left(2 \gamma_{r}^{2}\right)$, where $\tau=\omega_{p} t$ is the dimensionless time variable. This gives the following estimate for the energy transfer (in dimensionless units)

$$
\int_{0}^{T}\left(q_{b} \frac{\partial \phi}{\partial \xi}\left[\xi_{b}(\tau)\right]-\frac{q_{b}^{2}}{2}\right) d \tau=\gamma_{r}^{2}\left(2 q_{b} \Delta \phi-q_{b}^{2} \Delta \xi\right),
$$

with $\Delta \xi=\xi_{b}(T)-\xi_{b}(0)$ and $\Delta \phi=\phi\left[\xi_{b}(T)\right]-\phi\left[\xi_{b}(0)\right]$. The duration $T$ of the interaction is limited by phase slippage in the wakefield $\Delta \xi<\pi$, which limits the driver propagation distance to the dephasing length $\gamma_{r}^{2} \lambda_{p}$ (Reitsma et al. 2001), $\lambda_{p}=2 \pi c / \omega_{p}$ being the plasma wavelength. Given an initial condition $\xi_{b}(0)$ and the duration $T$ of the interaction, one may differentiate equation (4.5) with respect 
to $q_{b}$ to find the amount of charge that maximizes the energy transfer. This gives $q_{b}=\tilde{E}=\Delta \phi / \Delta \xi=\partial \phi / \partial \xi\left[\xi_{b}(\tilde{T})\right]$, where $\tilde{T} \in[0, T]$ is some intermediate time. The last formula implies that efficient energy transfer requires $q_{b}<E_{0}$, which is known as the beam loading limit. At $q_{b}=\tilde{E}$, the fraction of energy transferred from the wakefield to the bunch is $\tilde{E}^{2} / E_{0}^{2}$.

\section{(ii) Photons}

Now let the witness be an infinitely short laser pulse. As the magnitude of the wakefield excited by the photons depends on their frequency, let us first consider a monochromatic distribution, so that $f_{p}=N_{p} \delta\left[\xi-\xi_{m}(\tau)\right] \delta\left[k-k_{m}(\tau)\right]$ corresponds to a single macro-photon, where $N_{p}$ denotes the number of photons in the bunch and $\left(\xi_{m}, k_{m}\right)$ is the worldline of the macro-photon. The vector potential envelope is $a^{2}(\xi)=\omega_{p} N_{p} \delta\left(\xi-\xi_{m}\right) / \Omega$, so the magnitude of the jump of the electric field across the bunch, which we may use to define an equivalent charge $q_{p}$ (Mendonça et al. 1998) for the laser pulse, is equal to $q_{p}=\omega_{p} N_{p} /\left[2 \Omega(1+\phi)^{2}\right]$. As expected, the photon equivalent charge scales linearly with the number of photons, just like the electric charge is proportional to the number of charged particles. In addition, the equivalent charge also depends on the photon frequency and on the photon position in the wave. From the jump in the electric field, it is found that $\Delta \mathcal{C}=$ $\omega_{p} N_{p} \partial \phi / \partial \xi /\left[2 \Omega(1+\phi)^{2}\right]-\omega_{p}^{2} N_{p}^{2} /\left[8 \Omega^{2}(1+\phi)^{4}\right]$, with all quantities evaluated at $k=k_{m}, \xi=\xi_{m}$. Thus

$$
\frac{d \Omega}{d \tau}=-\frac{1}{2 \Omega} \frac{\partial \Omega_{p}^{2}}{\partial \xi}-\frac{\Omega_{p}^{8} N_{p}}{8 \omega_{p}^{5} \Omega^{2}},
$$

where the first term is the photon energy gain or loss in the prescribed field, while the second term represents the photon energy loss in its own wakefield. Net energy gain occurs if $\omega_{p} N_{p} / \Omega<(4 / 3) \partial(1+\phi)^{3} / \partial \xi$. As the photon distribution cannot be simultaneously localized in time and frequency, we must consider generalization to a pulse with finite bandwidth, which can be thought of as a collection of macrophotons with different $k_{m}$. For a distribution $f_{p}=\delta\left(\xi-\xi_{m}\right) \tilde{f}(k)$, the energy transfer to a macro-photon is

$$
\frac{d \Omega_{k}}{d \tau}=-\frac{1}{2 \Omega_{k}} \frac{\partial \Omega_{p}^{2}}{\partial \xi}-\frac{1}{8 \Omega_{k}} \frac{\Omega_{p}^{8}}{\omega_{p}^{5}} \int \frac{\tilde{f}\left(k^{\prime}\right)}{\Omega_{k^{\prime}}} d k^{\prime},
$$

where the pulse energy loss in its own wakefield is now seen to involve an integral over the $k$-distribution. Unfortunately, neither equation (4.6) nor equation (4.7) can be solved analytically. However, it is straightforward to check that the electron result is recovered if we assume that the photon initial frequency $\omega_{0}$ is large compared to the gain in frequency and the plasma wave amplitude is small. In this case, the equivalent charge $q_{p}$ becomes independent of the photon frequency and position, just like the electron bunch charge, and approaches $\omega_{p} N_{p} / 2 \omega_{0}$ (as $\Omega \approx \omega_{0}$ and $|\phi| \ll 1)$. Consequently, we may define the photon beam loading limit $q_{p}<E_{0}$, which is found from the energy transfer

$$
\gamma_{r}^{2}\left[\frac{\omega_{p} N_{p}}{\omega_{0}} \Delta \phi-\left(\frac{\omega_{p} N_{p}}{2 \omega_{0}}\right)^{2} \Delta \xi\right]=\gamma_{r}^{2}\left[2 q_{p} \Delta \phi-q_{p}^{2} \Delta \xi\right]
$$

where the photon velocity has been approximated with $c$, so that $d \xi_{m} / d \tau \approx 1 /\left(2 \gamma_{r}^{2}\right)$. 


\section{(b) Short driver evolution}

We now consider the feedback from the wake on a wakefield driver as it propagates in the plasma. The duration of the driver will be taken short as compared to a plasma period. For an infinitely short driver, we can apply the results from the previous subsection, as we may regard such a driver as a witness beam in a wakefield of zero amplitude.

\section{(i) Electrons}

As found above, the effective electric field on an infinitely short electron bunch due to its own wakefield is equal to $-q_{b} / 2$, where $q_{b}$ is the dimensionless bunch charge. Consequently, the bunch loses energy at a constant rate and is depleted at $\omega_{p} t=2 \gamma_{0} / q_{b}$, where $\gamma_{0}$ is the average initial Lorentz factor of bunch electrons. If the bunch length is finite, but still short compared to the plasma wavelength, the electric field varies continuously from 0 at the front of the bunch to approximately $-q_{b}$ at the rear. The consequence of the electric field variation is that electrons lose energy at different rates depending on their position within the bunch, which gives rise to a build-up of energy spread and, ultimately, to bunch lengthening due to velocity dispersion. The time at which bunch deformation becomes important is when the electrons in the rear of the bunch, whose energy loss rate is highest, have lost most of their energy, which is at $\omega_{p} t \approx \gamma_{0} / q_{b}$. It is assumed that the energy differences induced by the wakefield are large compared to the initial energy spread, so that velocity dispersion effects due to the latter can be neglected. Unless the bunch charge is very small $\left(q_{b} \gamma_{0} \ll 1\right)$, the energy depletion timescale is seen to be much shorter than the dephasing timescale for a witness bunch accelerated in the wakefield, whose phase velocity is estimated with $\gamma_{r} \approx \gamma_{0}$.

\section{(ii) Photons}

The interaction of a short laser pulse with its own wakefield leads to an explosive instability (Bulanov et al. 1992), as the drop in photon frequency increases the energy transfer rate from the laser pulse to the plasma. Taking a prescribed wake of zero amplitude, equation (4.6) reduces to $d \Omega / d t=-\omega_{p}^{4} N_{p} / 8 \Omega^{2}$, with solution $\Omega(t) / \omega_{0}=\left(1-t / t_{n l}\right)^{1 / 3}$, where $\omega_{0}$ is the initial frequency and $t_{n l}$ is the nonlinear energy depletion timescale, given by $\omega_{p} t_{n l}=\left(8 / 3 N_{p}\right)\left(\omega_{0} / \omega_{p}\right)^{3}=\left(4 / 3 q_{p}\right)\left(\omega_{0} / \omega_{p}\right)^{2}$. As in the case of an electron bunch, the electric field variation across a laser pulse with finite length makes the photon energy loss rate phase-dependent, which in turn leads to group velocity dispersion and laser pulse deformation. This effect becomes important when photons in the rear part of the laser pulse have lost most of their energy, i.e. when $t / t_{n l}=1-2^{1 / 2} / 4 \approx 0.65$. Estimating the wakefield phase velocity with $\gamma_{r} \approx \omega_{0} / \omega_{p}$, we find that the energy depletion timescale becomes comparable to the dephasing timescale of a witness beam at $q_{p}=q_{c r} \approx 3 \pi / 2$. For a laser drive pulse with a large amplitude, characterized by $q_{p} \gg q_{c r}$, the situation is identical to the case of an electron bunch driver, i.e. the depletion timescale is much shorter than the dephasing timescale. For a laser pulse with a small amplitude, characterized by $q_{p} \ll q_{c r}$, the depletion timescale is much longer than the dephasing timescale. In this case, the acceleration process is very inefficient in the sense that dephasing prevents the transfer of a large fraction of the driver energy to the witness. 


\section{Summary and discussion}

In this paper, we have made a comparison between the interaction of short laser pulses and relativistic electron bunches with plasma waves. Such a comparison is based on photon kinetic theory, i.e. a description of the laser field in terms of classical particles, which enables a direct comparison with the phase space evolution of the electrons. From the ray-tracing equations, one is able to define the equivalent of mass and energy for photons in plasma waves, which naturally leads to the concept of 'photon acceleration' in a plasma wave. By comparing the wakefields induced by short laser pulses and short electron bunches, one can define an effective charge for a photon in a plasma wave. This gives rise to a definition of photon beam loading, i.e. a limit on the number of photons that can be accelerated on a given plasma wave, analagous to electron beam loading.

We now comment on the differences between electrons and photons interacting with plasma. These differences stem from the qualitative difference between the ways in which photons and electrons excite plasma waves. As a measure of the strength of the interaction, we may use the jump in electric field across an infinitely short electron bunch or laser pulse, as calculated in $\S 4$. For electrons, the mechanism of plasma wave excitation is the Coulomb repulsion between bunch electrons and plasma electrons. The magnitude of the jump is proportional to the number of electrons in the bunch, irrespective of the bunch energy or its position in the plasma wave, as expected from Gauss' law. For photons, the magnitude of the jump becomes smaller at higher values of the photon frequency $\Omega$ or at lower values of $\Omega_{p}^{2}$. Both scalings can be understood by recalling that the mechanism of plasma wave excitation by a laser pulse is through the ponderomotive force. The ponderomotive force scales roughly with the vector potential envelope $a^{2} \propto N_{p} / \Omega$, which explains why, at the same number of photons $N_{p}$, the ponderomotive force is less effective at higher frequency. Also, the ponderomotive force is derived from the local plasma response to the high frequency electromagnetic oscillation (i.e. the quiver motion, described by $\left.\vec{p}_{\perp}=e \vec{A}_{\perp} / c\right)$. As $\Omega_{p}^{2}$ is nothing but the relativistically corrected local plasma electron density, the spatial dependence through $\Omega_{p}^{2}$ reflects that the plasma wave is excited less efficiently if fewer electrons participate in the quiver motion.

From the comparison of the wakefield excitation mechanisms, it is straightforward to explain the difference in the dynamics of photons and electrons in plasma waves. This is because the mechanism of energy transfer from a given plasma wave to the particles is beam loading, i.e. the excitation of a wakefield that (partly) cancels the given plasma wave, as shown in $\S 4$. The dependence of the equivalent mass, energy and charge of a photon in a plasma wave on its position in the wave stems from the dependence of its interaction on the local plasma electron density. The frequency dependence implies that photon acceleration in plasma waves is inefficient compared to electron acceleration, as the accelerating force decreases with increasing photon energy. This explains why different scalings for the maximum energy or frequency on the separatrix are found in $\S 3$ : the electron maximum energy, $2\left(1+\gamma_{r} \Delta \phi\right)$ in units of the resonant energy $\gamma_{r} m c^{2}$, is seen to be much larger than the photon maximum frequency, $2\left(1+\phi_{+}\right)^{1 / 2}$ in units of the resonant frequency $\gamma_{r} \omega_{p}$. The plasma wave amplitude thresholds for trapping counterpropagating photons or electrons from the bottom of the potential well (i.e. $k_{-}<0$ or $P_{-}<0$ ) are 
also seen to scale differently with $\gamma_{r}$. The fact that for electrons the threshold is much lower, rougly by a factor $2 \gamma_{r}^{1 / 2}$, emphasizes the point that electrons are, in a sense, 'easier to accelerate'.

Finally, we have discussed the interaction of a short plasma wave driver with its own wakefield, and we have found qualitatively different behaviour for electrons and photons. As the energy loss rate for photons depends on their frequency, the energy depletion from a short laser pulse accelerates and is explosively unstable. In contrast, the energy loss rate of a short electron bunch is approximately constant, as long as velocity dispersion effects can be neglected. By comparing the energy depletion timescale with the dephasing time of an accelerated witness beam, we have found that acceleration driven by an electron bunch is usually limited by the driver energy depletion. In contrast, dephasing is usually the limiting factor for acceleration driven by a laser pulse, unless the pulse has a high amplitude, i.e. if $\int a^{2}(\xi) d \xi \gg 1$.

\section{References}

Bulanov, S.V., Inovenkov, I.N., Kirsanov, V.I., Naumova, N.M. \& Sakharov, A.S. 1992 Nonlinear depletion of ultrashort and relativistically strong laser pulses in an underdense plasma. Phys. Fluids B 4, 1935-1942.

Chen, P., Dawson, J.M., Huff, R.W. \& Katsouleas, T. 1985 Acceleration of electrons by the interaction of a bunched electron beam with a plasma. Phys. Rev. Lett. 54, 693-696.

Esarey, E. \& Pilloff, M. 1995 Trapping and acceleration in nonlinear plasma waves. Phys. Plasmas 2, 1432-1436.

Esarey, E., Sprangle, P., Krall, J. \& Ting, A. 1996 Overview of plasma-based accelerator concepts. IEEE Trans. Plasma Sci. 24, 252-288.

Guérin, S., Laval, G., Mora, P., Adam, J.C., Héron, A. \& Bendib, A. 1995 Modulational and Raman instabilities in the relativistic regime. Phys. Plasmas 2, 2807-2814.

Keinigs, R. \& Jones, M.E. 1987 Two-dimensional dynamics of the plasma wakefield accelerator. Phys. Fluids 30, 252-263.

Mendonça, J.T. \& Silva, L.O. 1994 Regular and stochastic acceleration of photons. Phys. Rev. E 49, 3520-3523.

Mendonça, J.T., Silva, L.O., Bingham, R., Tsintsadze, N.L., Shukla, P.K. \& Dawson, J.M. 1998 Equivalent charge of photons and neutrinos in a plasma. Phys. Lett. A 239, 373-377.

Reitsma, A.J.W., Goloviznin, V.V., Kamp, L.P.J. \& Schep, T.J. 2001 Simulation of laser wakefield acceleration of an ultrashort electron bunch. Phys. Rev. E 63, 046502.

Silva, L.O. \& Mendonça, J.T. 1998 Kinetic theory of photon acceleration: time-dependent spectral evolution of ultrashort laser pulses. Phys. Rev. E 57, 3423-3431.

Tajima, T. \& Dawson, J.M. 1979 Laser electron accelerator. Phys. Rev. Lett. 43, 267-270.

Teychenné, D., Bonnaud, G. \& Bobin, J.L. 1994 Electrostatic and kinetic energies in the wake wave of a short laser pulse. Phys. Plasmas 1, 1771-1773.

Ting, A., Esarey, E. \& Sprangle, P. 1990 Nonlinear wake-field generation and relativistic focusing of intense laser pulses in plasmas. Phys. Fluids B 2, 1390-1394.

Wilks, S., Katsouleas, T., Dawson, J.M., Chen, P. \& Su, J.J. 1987 Beam loading in plasma waves. IEEE Trans. Plasma Sci. 15, 210-220. 